Braz. J. Aquat. Sci. Technol., 2010, 14(2):1-11.

\title{
PADRÕES ESPACIAIS E TEMPORAIS DE LARVAS DE SCARIDAE (PISCES: PERCIFORMES) NO NORDESTE DO BRASIL E SUAS RELAÇÕES COM OS FATORES OCEANOGRÁFICOS
}

\author{
SOUZA, C.S.*; BARREIRO, A. S. \& MAFALDA JR., P. \\ Universidade Federal da Bahia (UFBA) - Instituto de Biologia - Laboratório de Plâncton. 40210-020. \\ Ondina, Salvador/ BA - Brasil. \\ *Corresponding author: chsampaio@ig.com.br
}

\begin{abstract}
Souza, C.S.; Barreiro, A. S. \& Mafalda Jr., P. 2010. Spatial and temporal patterns of Scaridae larvae (Pisces: Perciformes) in the northeast of Brazil in relation to oceanographic conditions. Braz. J. Aquat. Sci. Technol. 14(2): 1-11. ISSN 1808-7035. This work had as objective to study the distribution and abundance of the Scaridae larvae and to analyze the influence of oceanographic conditions (temperature and salinity), on the space and temporal distribution of larvae. Ichthyoplankton was collected during expeditions: Period 1 (August - October 1995), Period 2 (January - April 1997), Period 3 (April - July 1998) and Period 4 ( September - December 2000), realized in the Northeast Exclusive Economic Zone. One specie (Cryptotomus roseus) and one genus (Sparisoma sp.) were identified. Sparisoma sp. was the most abundant genus ( $94 \%$ of the total), with higher abundance during all Periods. Cryptotomus roseus was the less abundant specie, representing $6 \%$ of the total identified larvae. Cryptotomus roseus presented larger distribution with the maximum density during period 3, between Maceió and Aracajú. Sparisoma sp. larvae were found mainly in oceanic stations and near São Pedro and São Paulo Archipelago.
\end{abstract}

Keywords: Ichthyoplankton, Scaridae, distribution, abundance.

\section{INTRODUÇÃO}

Os peixes da família scaridae, conhecidos como peixe-papagaio, compreendem um dos muitos grupos predominantes de peixes que habitam mares tropicais e recifes de corais, possuem aproximadamente 90 espécies distribuídas em 10 gêneros (Richards, 2006).

$\mathrm{Na}$ região central oeste do Atlântico Norte quatorze espécies ocorrem em quatro gêneros. Dois gêneros, Sparisoma e Cryptotomus, são endêmicos do Atlântico (Richards, 2006). Quatro gêneros de Scaridae são registrados no sudoeste Atlântico, Cryptotomus, Nicholsina, Scarus e Sparisoma (Carvalho-Filho 1999; Humann \& Deloach 2002). Sparisoma é o gênero com maior quantidade de espécies nessa região (Bernardi et al., 2000; Streelman et al., 2002). Este gênero é ecologicamente diverso, apresentando uma extensa gama de formas alimentares e padrões de uso de hábitat (Bernardi et al., 2000; Streelman et al., 2002; Ferreira et al., 1999). Larvas de Scaridae não foram registradas em áreas estuarinas do nordeste brasileiro (Mafalda \& Silva 1996; Ekau et al., 2001; Marcolin et al., 2010), indicando que ela crescem em zonas oceânicas e de plataforma.

Pouco se sabe sobre abundância larval e padrões de distribuição da família scaridae no Brasil. Recente estudo realizado na Zona Econômica Exclusiva do Nordeste fez um levantamento de informação relevante sobre a ocorrência dessa família. Algumas espécies comercialmente importantes são encontradas no litoral nordestino, destacando-se os peixes das espécies
Sparisoma rubripinne (Valenciennes, 1839), Sparisoma viride (Bonaterre, 1788), Sparisoma chrysopterum (Bloch e Schneider, 1801) e Scarus coelestinus (Valenciennes, 1839) (Lessa et al., 2000). Essas espécies são conhecidas respectivamente pelos nomes vulgares: Bobó, budião, budião azul e bico verde ou papagaio

As larvas de peixe são espacialmente e temporalmente dinâmicas e as variações na composição e abundância ocorrem verticalmente e horizontalmente em uma escala de tempo (Leis, 1991). Processos múltiplos contribuem para essa variabilidade e podem incluir as atividades de desovas dos peixes adultos (Nonaka et al., 2000), mudanças de desenvolvimento na capacidade de uma larva individual (Leis \& Mccormick, 2002), e aspectos do ambiente oceanográfico que a larva habita (Cowen, 2002).

Em regiões temperadas, padrões de distribuição e abundância de larvas de peixe tem sido objeto de pesquisa por décadas. Em contraste, há poucos estudos em ambientes tropicais (Sampey et al., 2004), examinando padrões temporais. Muitos trabalhos nos trópicos têm focado em padrões espaciais, tanto em pequena escala (dezenas de $\mathrm{km}$ ) próximo a recifes de corais (Leis \& Goldman, 1987) como em larga escala (centenas a milhares de $\mathrm{km}$ ) através de plataformas continentais (Young et al., 1986) e oceanos (Ahlstrom, 1972).

Alterações nas condições ambientais podem resultar em mudanças na estrutura da comunidade 
devido ao efeito que as mesmas teriam na distribuição, abundância e composição do ictioplâncton (Whitfield \& Paterson, 2003). Uma boa compreensão destas interações é importante para a conservação dos sítios de desova e criação das larvas de peixe, bem como propiciam um melhor entendimento da variabilidade no recrutamento dos recursos pesqueiros.

Este estudo analisou os padrões espaciais e temporais de larvas de Scaridae e avaliou a influência de fatores oceanográficos (temperatura, salinidade, biomassa fitoplanctônica e biomassa zooplanctônica), sobre a densidade das larvas na Zona Econômica Exclusiva do Nordeste do Brasil.

\section{ÁREA DE ESTUDO}

A área de estudo corresponde à região Nordeste da ZEE-brasileira (ZEE/NE), abrangendo a coluna d'água até a profundidade de $200 \mathrm{~m}$, desde o limite das 12 milhas náuticas (MN) do mar territorial brasileiro até o contorno distando $200 \mathrm{MN}$ da linha de costa do Brasil e em volta do arquipélago de São Pedro e São Paulo, limitada ao sul pelo paralelo que passa por Salvador (BA) e a norte pelo meridiano que corta a foz do rio Parnaíba (PI), totalizando $2.000 \mathrm{Km}$ de linha de costa e uma extensão de $1.450 .000 \mathrm{Km}^{2}$ (Madeiros et al., 2009a).

A região apresenta um perfil regular, o qual é quebrado nos extremos norte e sul por estuários de grandes rios, destacando-se o Parnaíba (PI) e o São Francisco (SE). A barreira de recifes é uma característica notável da costa especialmente entre Natal e Aracaju. Adicionalmente, vários grupos de ilhas e rochedos são aí encontrados - Atol das Rocas, arquipélago de Fernando de Noronha e arquipélago de São Pedro e São Paulo. Além das ilhas oceânicas, ao largo da plataforma continental, observa-se uma série de bancos rasos, pertencentes às cadeias Norte-brasileira e de Fernando de Noronha, notadamente em frente aos estados do Ceará e Rio Grande do Norte. A maior parte do domínio oceânico é formada por áreas de grande profundidade, entre 4.000 e $5.000 \mathrm{~m}$, as quais correspondem às Planícies abissais do Ceará e de Pernambuco (Figura 1). A plataforma continental apresenta-se pouco profunda, com seu limite externo situado entre as isóbatas de 40 e 80 m (REMAC, 1975).

A ZEE Nordeste é dominada pelas correntes resultantes da bifurcação da Corrente Sul Equatorial - a Corrente Norte do Brasil, que segue rumo às Guianas, e a Corrente do Brasil, que flui para o sul (Evans \& Signorini, 1985). A maior intensidade dos ventos alísios de Sudeste, no segundo semestre do ano, resulta de intensificação do sistema equatorial de correntes, notadamente da Contracorrente Norte Equatorial e da

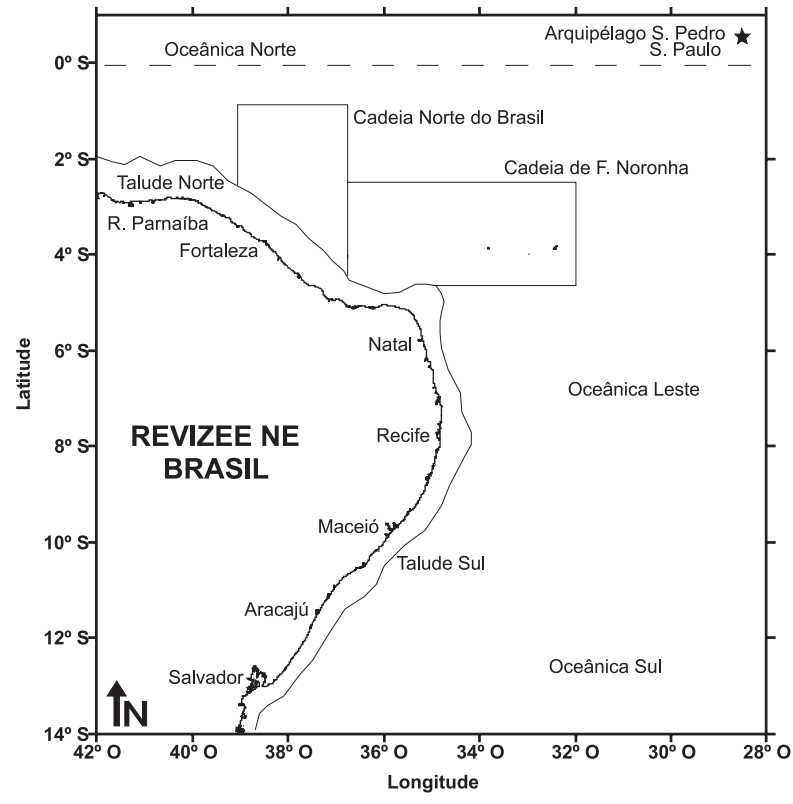

Figura 1 - Área de estudo na ZEE Nordeste do Brasil.

Corrente Equatorial Submersa, as quais praticamente inexistem a oeste de $20^{\circ} \mathrm{S}$, durante o primeiro semestre do ano (Evans \& Signorini, 1985).

\section{MATERIAL E MÉTODOS}

As amostragens foram feitas durante as expedições REVIZEE Nordeste I, II, III e IV, realizadas entre 1995 - 2000, nos seguintes períodos: Nordeste I-02/ Agosto a 26/Outubro de 1995 (Período 1); Nordeste II 21/Janeiro a 13/Abril de 1997 (Período 2); Nordeste III 28/Abril a 20/Julho de 1998 (Período 3) e Nordeste IV 25/setembro a 06/dezembro de 2000 (Período 4). Neste estudo foram utilizadas as 562 amostras obtidas durante as quatro expedições realizadas na ZEE Nordeste (Figura 2).

O ictioplâncton foi coletado segundo metodologia proposta por Smith \& Richardson (1977), através de rede do tipo Bongo com abertura de $50 \mathrm{~cm}$, malhas de 300 e $500 \mu \mathrm{m}$, dotadas de fluxômetros (Hydro-Bios) para o cálculo do volume de água filtrada. Neste estudo foi analisado apenas o ictioplâncton contido na rede de $500 \mu \mathrm{m}$. Os arrastos oblíquos foram realizados variando de acordo com a profundidade, a partir dos 5 metros do fundo em estações rasas, e 200 metros de profundidade nas demais estações, com duração média de 10 minutos.

A triagem total de larvas e jovens de Scaridae e sua identificação ao menor nível taxonômico possível foram realizadas por meio de microscópio estereoscópico Wild MZ6. Para a identificação dos gêneros e espécies da família scaridae, foram empregados os trabalhos de Leis \& Remmis (1983), Richards 


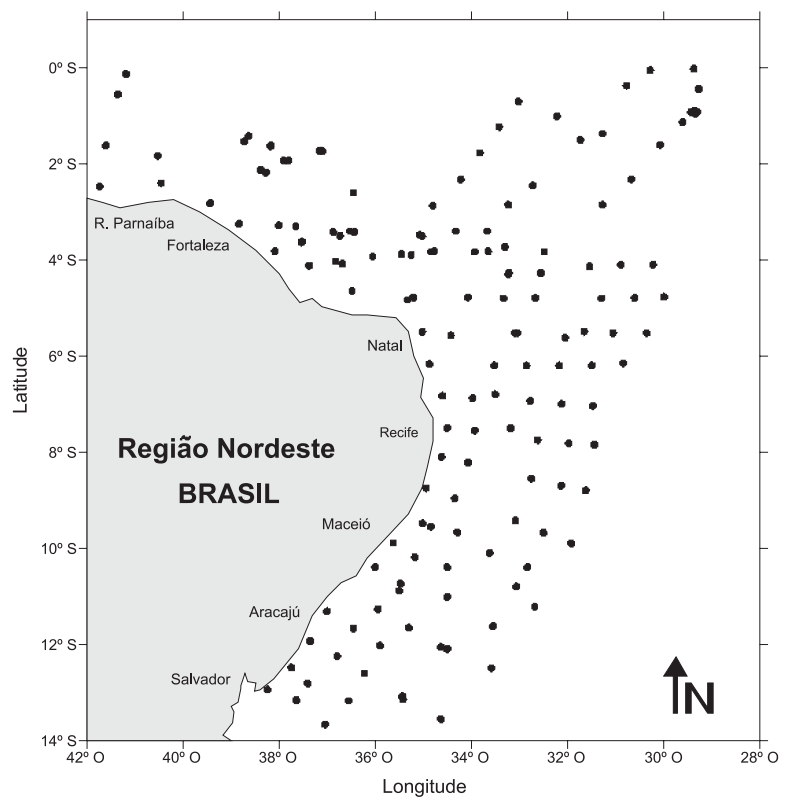

Figura 2 - Estações de amostragem na ZEE Nordeste do Brasil.

et al., (2006). A técnica de identificação envolveu a análise de caracteres morfológicos (formato do corpo), morfométricos (comprimento padrão) e merísticos (padrão pigmentar). As larvas de Sparisoma sp. foram identificadas no nível genérico pois para realizar a identificação das espécies seria necessária uma análise de DNA (Richards, 2006). A densidade de larvas de peixe foi estimada por $100 \mathrm{~m}^{3}$ de água filtrada.

A caracterização da massa de água foi realizada através de registros de salinidade e de temperatura $\left({ }^{\circ} \mathrm{C}\right)$, obtidos através do CTD. As amostras de água destinada às análises de biomassa fitoplanctônica a $1 \%$ de luz (clorofila a), foram obtidas por meio de garrafas de Ninski. A análise de clorofila a foi realizada através do método espectrofotométrico de Strinckland \& Parsons (1972). A determinação da biomassa zooplanctônica (peso seco - rede de $500 \mu \mathrm{m}$ ) foi realizada segundo metodologia de Omori \& Ikeda (1984).

\section{Análises dos dados}

Visando investigar a ocorrência de variabilidade temporal nos dados abióticos e bióticos (densidade das espécies) foi aplicado o teste não paramétrico de Kruskal - Wallis, seguido do teste de comparações múltiplas de Dunn, com nível de significancia de 5\%.

A representação da distribuição espacial da densidade de larvas de peixes foi realizada através da elaboração de mapas geo-referenciados. A caracterização das massas de água foi feita através do diagrama TS com dados de superfície.

As flutuações na densidade dos taxa da família scaridae, durante os quatro períodos estudados, foram relacionadas com temperatura e salinidade através do diagrama TSD (Temperatura, Salinidade e Densidade) e da análise de correlação.

\section{RESULTADOS}

\section{Hidrologia}

A distribuição horizontal da temperatura do mar à superfície oscilou entre 26,1 e $27,8^{\circ} \mathrm{C}$ (média=26,6 ${ }^{\circ} \mathrm{C}$ ) durante o período 1 , entre 26,2 e $29,4^{\circ} \mathrm{C}$ (média $=28,9$ ${ }^{\circ} \mathrm{C}$ ), durante o período 2 , entre 25,8 e $28,6^{\circ} \mathrm{C}$ (média $=27,1^{\circ} \mathrm{C}$ ), durante o período 3 e entre 25,7 e $28,4^{\circ} \mathrm{C}$ (média $=26,8$ ), durante o período 4 (Figura 3). Verificou-se uma elevação global da temperatura das águas superficiais com cerca de $1,5^{\circ} \mathrm{C}$, entre os períodos 1 e 2 . A temperatura apresentou uma diferença extremamente significante entre os quatro períodos analisados (Kruskal-Wallis, $p<0,0001$ ), comprovando a ocorrência de variação temporal. O teste de comparações múltiplas de Dunn verificou que o período 1 e o período 4 , diferem do período 2 e do período 3.

A salinidade nas camadas mais superficiais, oscilou entre 35,49 e 37,24 (média $=36,1$ ), durante o período 1, entre 34,8 e 37,2 (média $=36,2$ ), durante o período 2, entre 34,5 e 37,4 (média=36,4), durante 0 período 3 e entre 35,2 e 37,2 (média=36,3), durante o período 4 (Figura 4). O resultado do teste de KruskalWallis para salinidade demonstrou uma diferença extremamente significante entre os quatro períodos analisados (Kruskal-Wallis, $p<0,0001$ ), comprovando a ocorrência de variação temporal. O teste de comparações múltiplas de Dunn verificou que o período 1 e o período 2, diferem do período 3 e do período 4.

Os valores de salinidade e temperatura, registrados em todos os períodos investigados, indicaram a predominância da Água Tropical Superficial (ATS), também conhecida como Água Equatorial Superficial (AES). Em raros momentos detectou-se uma massa de água Costeira $(A C)$, com salinidade em torno de 35 (Figura 5).

\section{Biomassa Planctônica}

\section{Biomassa fitoplanctônica}

No nível de $1 \%$ de intensidade de luz a concentração de clorofila a apresentou valores extremamente baixos no período 1, que oscilaram entre 0,03 e 0,2 $\mu \mathrm{g} /$ L. Valores intermediários variando entre 0,2 e 3,9 $\mu \mathrm{g} / \mathrm{L}$, foram encontrados no período 2 . Os valores mais elevados foram obtidos nos períodos 3 e 4 , onde oscilaram entre 0,1 e $5,1 \mu \mathrm{g} / \mathrm{L}$, e entre 0,2 e $5,9 \mu \mathrm{g} / \mathrm{L}$, respectivamente (Figura 6).

O resultado do teste de Kruskal-Wallis demonstrou uma diferença extremamente significante entre os quatro períodos analisados (Kruskal-Wallis, $p<0,0001$ ), comprovando a ocorrência de variação temporal. Con- 

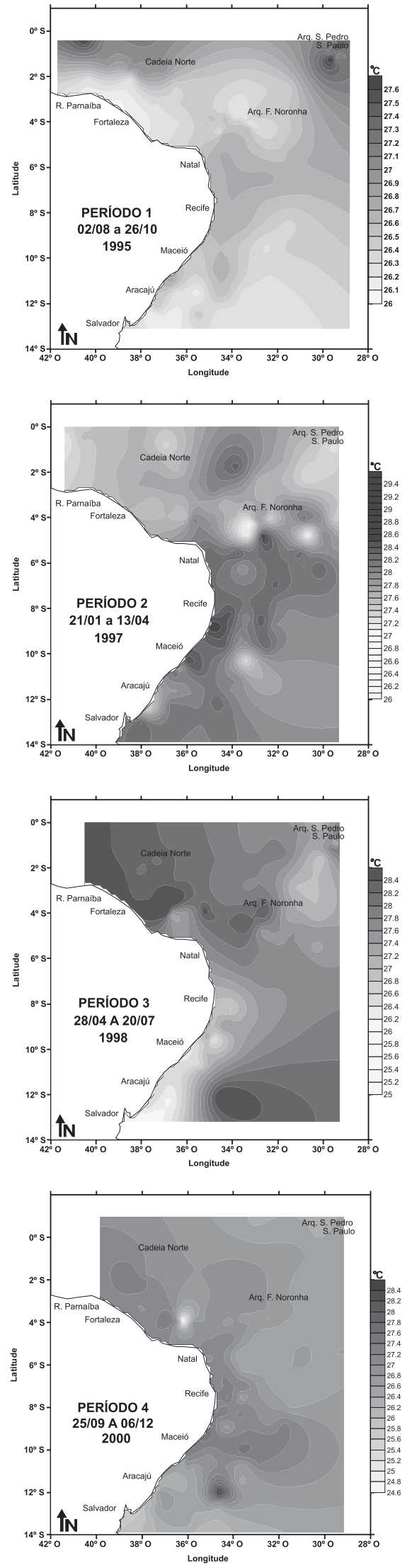

Figura 3 - Distribuição espacial e temporal da temperatura para os quatro períodos estudados.
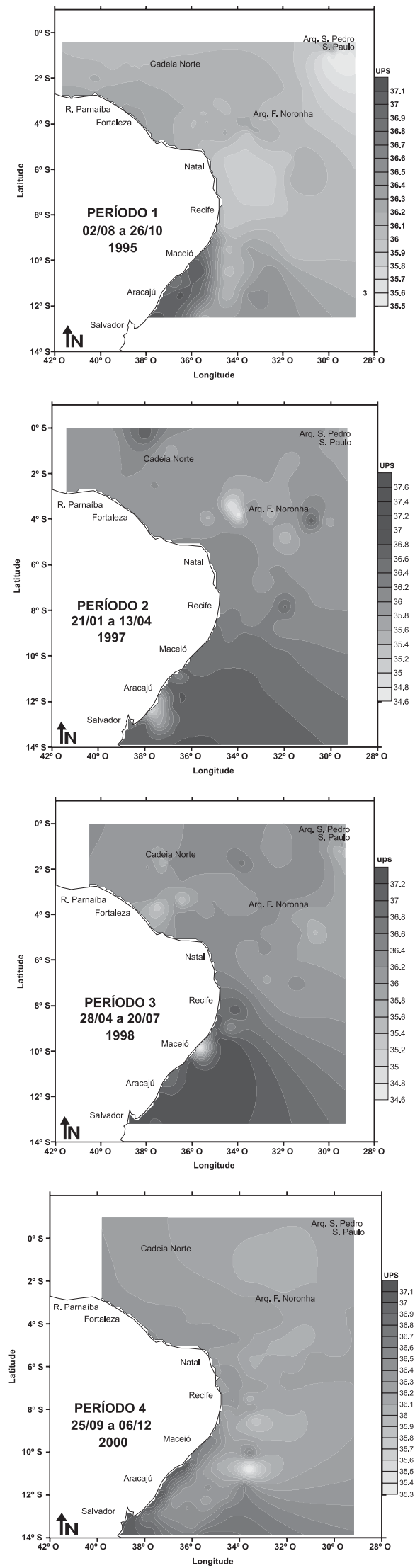

Figura 4 - Distribuição espacial e temporal da salinidade para os quatro períodos estudados. 
PERÍODO 1

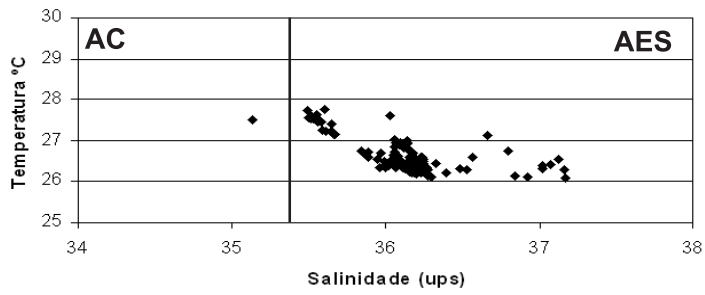

PERÍODO 2

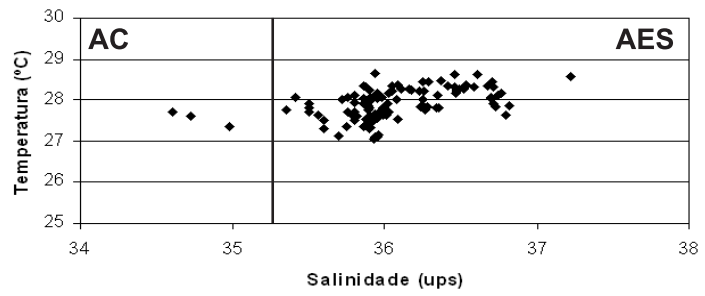

PERÍODO 3

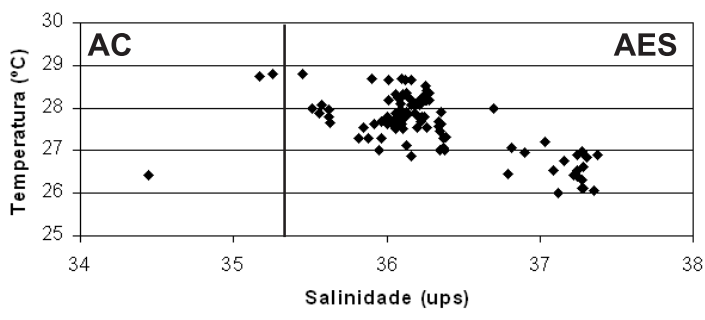

PERÍODO 4

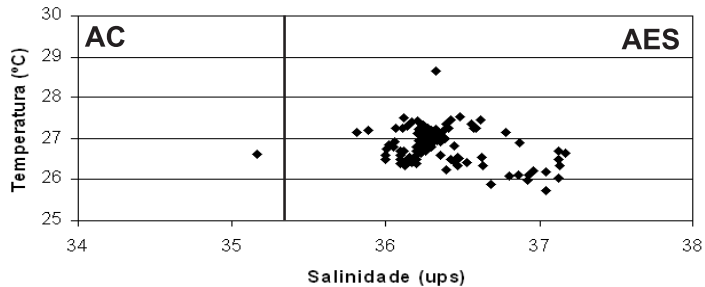

Figura 5 - Diagrama T/S para os quatro períodos investigados.

tudo, o teste de comparações múltiplas de Dunn constatou que esta diferença não ocorreu apenas entre os períodos 3 e 4.

\section{Biomassa zooplanctônica}

O peso seco apresentou valores mais baixos no Período 1 oscilando entre 0,02 e 1,6g/100 $\mathrm{m}^{3}$ e no Período 4 , que oscilaram entre 0,2 e $2,4 \mathrm{~g} / 100 \mathrm{~m}^{3}$. Valores intermediários variando entre 0,07 e $4,1 \mathrm{~g} / 100 \mathrm{~m}^{3}$, foram encontrados no Período 3. Os valores mais elevados foram obtidos no Período 2, onde oscilaram entre 0,03 e $11,3 \mathrm{~g} / 100 \mathrm{~m}^{3}$. Nos 4 períodos investigados, os valores de biomassa zooplanctônica tenderam a aumentar nas áreas adjacente à Cadeia Norte do Brasil, Arquipélago de São Pedro São Paulo e Cadeia de Fernando de Noronha (Figura 7).
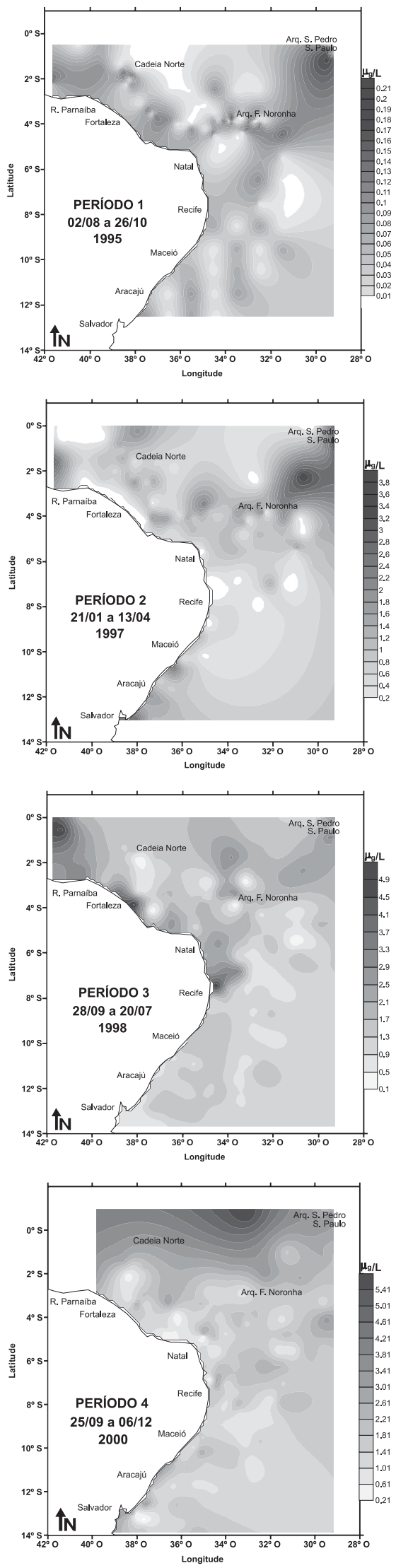

Figura 6 - Distribuição espacial e temporal da biomassa fitoplanctônica (Clorofila a), para os quatro períodos estudados. 
Para o peso seco, obtido com a rede de $500 \mu \mathrm{m}$, o resultado do Teste de Kruskal-Wallis, demonstrou uma diferença extremamente significante entre os quatro períodos analisados (Kruskal-Wallis, $p<0,0001$ ), comprovando também a ocorrência de variação temporal. $O$ teste de comparações múltiplas de Dunn constatou que o período 1 difere dos períodos 2, 3 e 4 .

\section{Composição e ocorrência de larvas de Scaridae}

Em um total de 658 larvas foram identificados uma espécie (Cryptotomus roseus) e um gênero (Sparisoma sp.) pertencentes à família scaridae, ocorrendo na ZEE Nordeste.

O Gênero Sparisoma sp. foi o taxon dominante na ZEE nordeste, representando $94 \%$ do total de larvas identificadas. As abundâncias elevadas foram observadas durante todos os períodos, sendo os Períodos 1 e 4 maiores que os Períodos 3 e 2. Cryptotomus roseus representou apenas $6 \%$ do total de scarídeos. Esta espécie teve sua maior abundância durante o Período 3, sendo o Período 1 e Período 4 os de menor abundância (Figura 8).

Os dois taxa ocorreram em todos os períodos estudados. Sparisoma sp. apresentou os valores mais elevados de freqüência de ocorrência, sendo o período 3 o de maior freqüência $(28,67 \%)$ e o período 1 o de menor (14,81\%). Cryptotomus roseus, ao contrário, teve baixa freqüência em todas as épocas porém, também no período 3 , apresentou a maior freqüência (10\%), sendo o período 4 o de freqüência extremamente baixa $(0,82 \%)$ (Figura 9).

A densidade das espécies também apresentou diferença significante entre os quatro períodos analisados (Kruskal-Wallis, $p<0,01$ ), comprovando a ocorrência de variabilidade temporal.

\section{Distribuição espacial e temporal}

As larvas da família Scaridae foram coletadas mais freqüentemente dentro da zona oceânica do Nordeste do Brasil nas áreas da Cadeia Norte do Brasil, Cadeia de Fernando de Noronha, Oceânica Leste e Oceânica Sul.

A espécie Cryptotomus roseus apresentou uma distribuição por toda a área estudada, principalmente durante o período 3 , onde esteve presente desde locais adjacentes à costa (próximo a Maceió), até locais mais profundos, ocorrendo inclusive na região oceânica e nas áreas de bancos e ilhas do nordeste (Cadeia Norte do Brasil, Cadeia de Fernando de Noronha e Arquipélago de São Pedro São Paulo). Observou-se que $C$. roseus ocorreu nos quatro períodos estudados, entretanto, durante o período 4, a espécie ocorreu apenas em uma estação de amostragem. As densidades variaram de 0,01 a 5 larvas $/ 100 \mathrm{~m}^{3}$ para todas as épocas. Contudo, a maiores densidades foram registradas durante os
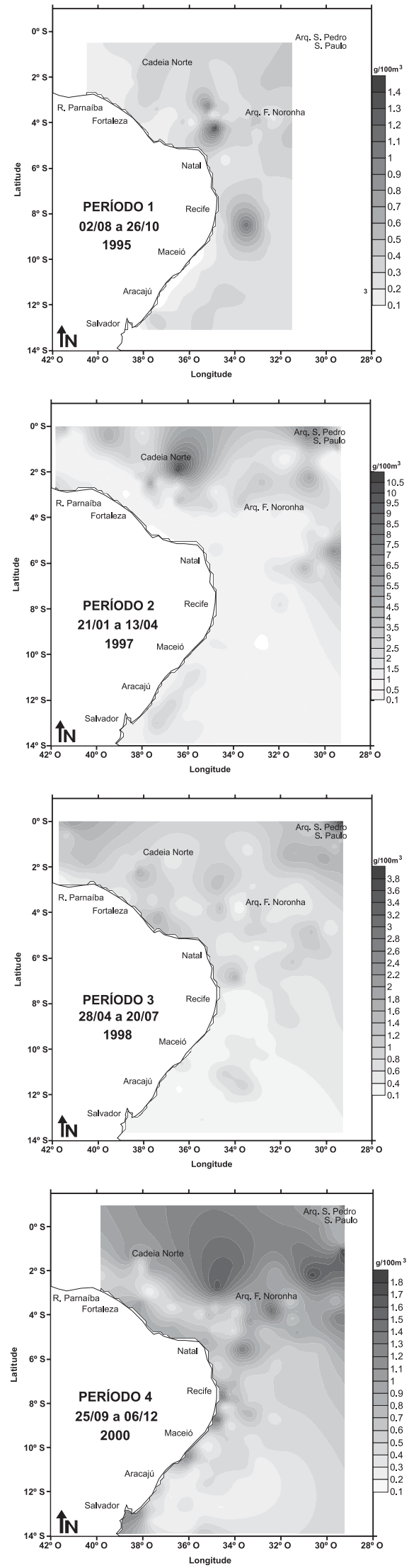

Figura 7 - Distribuição espacial e temporal da biomassa zooplanctônica (peso seco) para os quatro períodos estudados. 


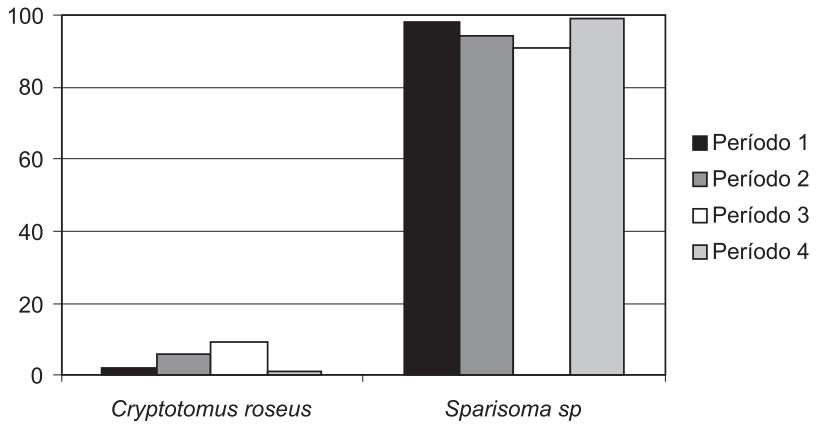

Figura 8 - Abundância relativa de Scaridae para os quatro períodos investigados.

períodos 2 e $3(2,21$ e 4,56 larvas/100m³ respectivamente) (Figura 10).

O gênero Sparisoma sp. apresentou uma ampla distribuição, ocorrendo também nos quatro períodos. Durante o período 1 sua ocorrência foi principalmente em estações localizadas nas regiões próximas à $\mathrm{Ca}$ deia Norte do Brasil e ao Arquipélago de Fernando de Noronha, sendo que as maiores densidades ocorreram na região Oceânica Leste. Os períodos 2 e 3 foram às épocas em que o gênero esteve presente em um maior número de estações amostradas. Ocorrendo na área do Talude Sul (entre Natal e Aracajú), na área Oceânica Leste e de bancos e ilhas, tendo suas maiores densidades registradas próximo ao Arquipélago de Fernando de Noronha durante o período 2 e na área Oceânica Leste e São Pedro São Paulo durante o período 3. Durante o período 4 os valores mais elevados de densidade foram observados na região Oceânica Sul (Figura 10).

\section{Relação entre larvas de Scaridae e fatores oceanográficos}

A análise de correlação entre a densidade de larvas de Sparisoma sp. e as variáveis oceanográficas analisadas (temperatura e salinidade) não apresentou diferença significativa $(p=0,64)$. Nenhum padrão foi observado no diagrama TSD (Figura 11).

Para Cryptotomus roseus a correlação entre a densidade de larvas e a temperatura e salinidade foi significante $(p=0,03)$, onde ficou evidente que a temperatura $(p=0,02)$ e a salinidade $(p=0,04)$, contribuíram para a variabilidade da espécie. A temperatura e a salinidade influenciaram positivamente a densidade de Cryptotomus roseus.

O diagrama TSD revelou que a maioria das ocorrências esteve associada a temperaturas superiores à $27^{\circ} \mathrm{C}$ e a salinidades maiores de 36 (Figura 11), ou seja, na presença de uma Água Tropical Superficial (Medeiros et al., 2009b). Essa massa d'água é transportada para o sul pela Corrente do Brasil, ocupando a camada mais superficial do oceano (200 m superiores).

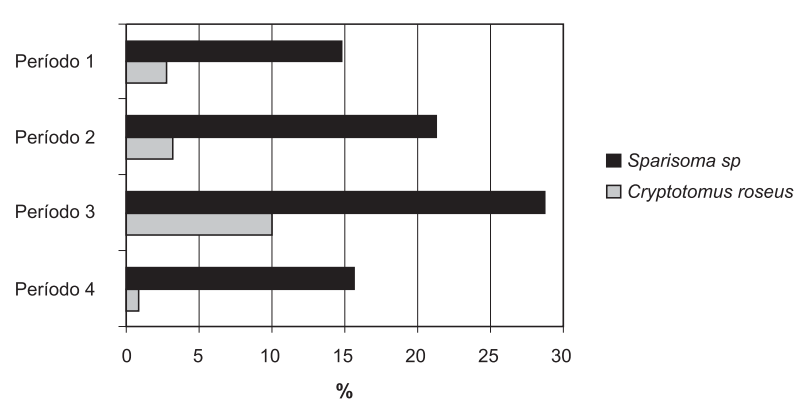

Figura 9 - Freqüência de ocorrência de Scaridae para os quatro períodos investigados.

\section{DISCUSSÃO}

O ambiente oceanográfico da ZEE/NE é dominado pelo sistema de correntes Sul Equatorial, que flui entre os paralelos $10^{\circ}$ e $25^{\circ}$ S em direção à costa Brasileira próxima da qual bifurca-se na Corrente Norte do Brasil e a Corrente do Brasil (Stramma, 1991; Reid, 1989), que originam dois ambientes oceanográficos distintos ao longo da porção oceânica estudada. De acordo com os resultados de temperatura e salinidade, a área de estudo apresentou características típicas de massa de água Tropical Superficial (ATS), com salinidade e temperaturas elevadas e pequena oscilação térmica. De acordo com Medeiros et al. (2009b) a massa de água ATS ocupa a camada superficial da ZEE/NE (primeiros 75-215m), apresentando menor espessura na região oceânica sul e maior na região do Arquipélago de São Pedro e São Paulo. Becker (2001) afirmou que a profundidade de salinidade máxima na Cadeia Norte do Brasil e Arquipélago de Fernando de Noronha, normalmente ocorre na faixa de $1 \%$ de luminosidade ou no início da termoclina, entre 50-100 m, sendo mais superficial no Arquipélago de São Pedro São Paulo.

As maiores concentrações de clorofila a foram encontradas no período 3 e período 4 e os menores valores no período 1 e período 2 . O aumento temporal da biomassa fitoplanctônica, pelo efeito do enriquecimento dos nutrientes, em águas oligotróficas tropicais também foi registrado no Mar de Sargassos (Menzel \& Ryther, 1961), Mar Arábico (Rhyter \& Menzel, 1965), Oceano Pacífico Leste (Owen \& Zeitschel, 1970) e no Oceano Pacífico Nordeste (Thomas, 1966).

Nos quatro Períodos investigados, os valores de macrozooplâncton tenderam a aumentar nas áreas adjacentes a Cadeia Norte do Brasil, Arquipélago de Fernando de Noronha e Arquipélago de São Pedro São Paulo, possivelmente devido à ressurgências topográficas (Carvalho et al., 2006). Em um banco oceânico na cadeia Norte Brasileira, um pronunciado cone de ressurgência foi registrado, sugerindo a formação de uma coluna de Taylor, que apresenta um grande poten- 

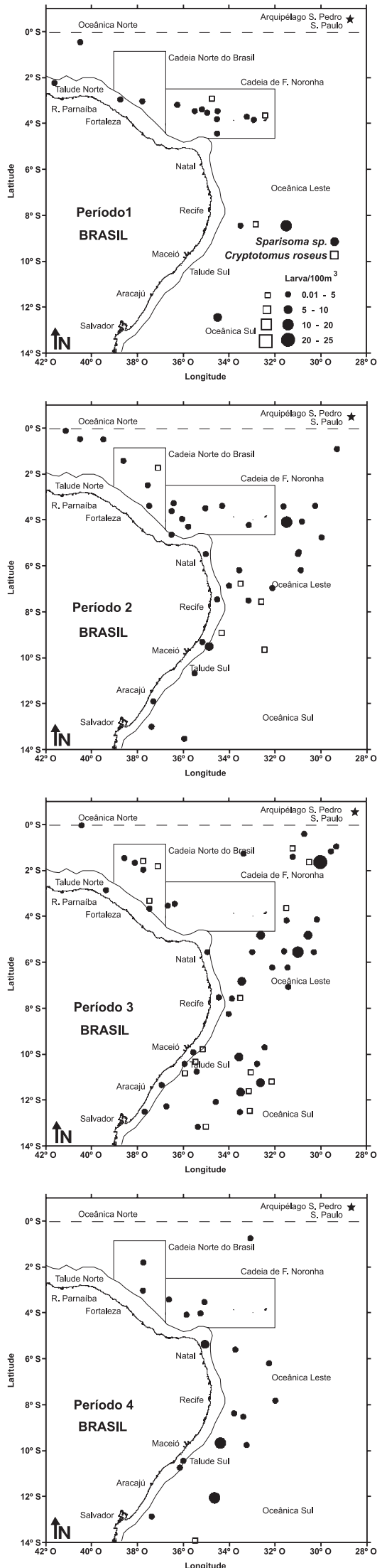

Figura 10 - Distribuição espacial e temporal de Sparisoma sp. e Cryptotomus roseus $/ 100 \mathrm{~m}^{3}$, na ZEE Nordeste. cial para o enriquecimento das camadas superiores (Travassos et al., 1999). Valores mais elevados de biomassa zooplanctônica também foram registrados, durante o Período 4, na quebra de plataforma e talude leste, provavelmente devido a ressurgência de quebra de plataforma. De acordo com Medeiros et al (1999), a ressurgência de quebra de plataforma esta entre os mecanismos que contribuem para o aumento da produtividade planctônica em águas do Nordeste Brasileiro. Não obstante, em outros estudos realizados na plataforma entre Ceará e Pernambuco, a biomassa mesozooplanctônica $\left(12,14-117,14 \mathrm{mg} / 100 \mathrm{~m}^{3}\right)$ e a biomassa macrozooplanctônica $\left(<50 \mathrm{mg} / \mathrm{m}^{3}\right)$ foram muito baixas (Ekau et al., 1999; Neumann-Leitão et al., 1999), e nas isóbatas entre 200 e $1000 \mathrm{~m}$, onde foram observadas concentrações mais elevadas estas também foram atribuídas a picos de ressurgência causados pela interação entre a ACAS e o talude continental (Ekau et al., 1999). Boltovskoy (1981) menciona que a biomassa máxima zooplanctônica no Atlântico Sul Ocidental ocorre nas águas neríticas com valores atingindo $100 \mathrm{mg} \cdot \mathrm{m}^{-3} \mathrm{na}$ camada de $200 \mathrm{~m}$.

Não houve mudança na composição de Scaridae durante todos os períodos estudados, no entanto podese observar a existência de períodos preferências, como por exemplo, os períodos 2 e 3 onde foram encontrados os maiores valores de abundância e freqüência de ocorrência, tanto para Sparisoma sp. quanto para Cryptotomus roseus principalmente na região oceânica e nas áreas de bancos e ilhas do nordeste (Cadeia Norte do Brasil, Cadeia de Fernando de Noronha e Arquipélago de São Pedro São Paulo).

No Atlântico Sul Ocidental ocorrem espécies de peixe-papagaio dos gêneros Cryptotomus, Nicholsina, Scarus e Sparisoma. As espécies Sparisoma viride, Sparisoma rubripinne e Sparisoma chrysopterum ocorrem do Maranhão a Santa Catarina e no Arquipélago de Fernando de Noronha, Pernambuco (Moura et al., 2001). Foram observados também no Sudeste do Caribe (Bonaldo, 2006). As espécies Sparisoma viride, Sparisoma rubripinne e Sparisoma chrysopterum estão entre as espécies de peixe papagaio mais abundantes em recifes rasos do Atlântico Ocidental (CarvaIho-Filho, 1999).

Larvas de $C$. roseuse Sparisomasp. tiveram suas maiores densidades em águas mais quentes e mais salinas. Houve uma variação na distribuição e abundância, tanto espacial como temporal e possivelmente no ciclo reprodutivo dos taxa de Scaridae.

Variações no ambiente oceanográfico, em uma escala anual, podem causar mudanças interanuais na distribuição dos peixes adultos e nas características de seu ambientes de desova, assim como, duração e localização de desova (Doyle et al., 1993). Assim, a distribuição de larvas tem sido examinada dentro de 

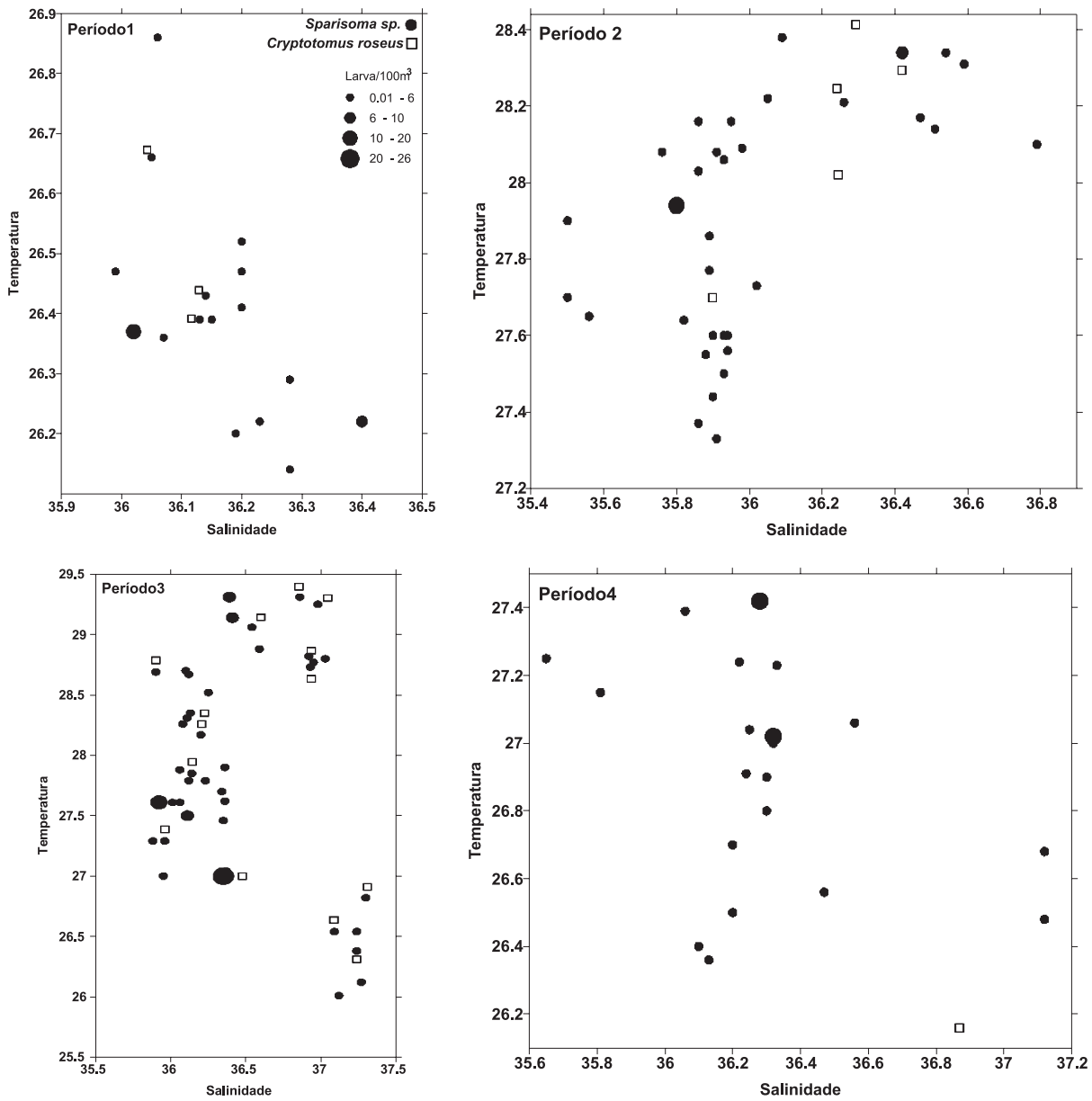

Figura 11 - Diagrama TSD para os quatro períodos investigados.

uma estrutura dinâmica, pois as condições hidrográficas simplesmente não têm explicado significativamente a variabilidade na distribuição de assembléias ou o processo de recrutamento (Govoni, 1993; Cowen et al., 1993; Dempserter et al., 1999).

\section{CONCLUSÕES}

Os padrões espaciais e temporais de larvas de Scaridae foram diferentes em todos os períodos estudados. Temperatura e salinidade influenciaram nas densidades das larvas de Cryptotomus roseus, no entanto, as densidades das larvas de Sparisomasp. não foram influenciadas pelos fatores oceanográficos (temperatura e salinidade).

\section{AGRADECIMENTOS}

À Comissão Interministerial dos Recursos do Mar, Ministério do Meio Ambiente, Sub-Comitê Regional REVIZEE-Nordeste, Diretoria de Hidrografia e Navega- ção e Navio Oceanográfico ANTARES, pelo apoio que possibilitou o desenvolvimento deste trabalho. A FAPESB, pela possibilidade de desenvolver em período integral as atividades de pesquisa através da bolsa de auxílio ao programa do Mestrado em Ecologia e Biomonitoramento da UFBA.

\section{REFERÊNCIAS BIBLIOGRÁFICAS}

Becker, M. 2001. Hidrologia dos Bancos e llhas oceânicas do Nordeste Brasileiro, uma contribuição ao Programa Revizee. Tese de Doutorado. Universidade Federal de São Carlos. 151p.

Bernardi, G.; Robertson, D. R; Clifton, K. \& Azzurro, E. 2000. Molecular systematics, zoogeography, and evolutionary ecology of the Atlantic genus Sparisoma. Molecular Phylogenetic Evolution. 15:292-300.

Boltovskoy, D. 1981. Atlas del Zooplancton del Atlántico Sudoccidental y métodos de trabajo con el zooplancton marino. INIDEP, Mar del Plata, 936p. 
Bonaldo, R. M. 2005. Atividade de forrageamento de três espécies sintópicas de Sparisoma (Perciformes: Scaridae) no arquipélago de Fernando de Noronha, Pernambuco. Dissertação de Mestrado. Universidade Estadual de Campinas. 132p.

Carvalho, T. B.; Mafalda Júnior, P.; Santos, C.; Souza, C.S.; Moura, G.; Sampaio, J.; Melo, G.; Passavante, J. Z. O. \& Feitosa, F. A. N. 2006. Biomassa planctônica e hidrografia na Zona Econômica Exclusiva do Nordeste do Brasil. Tropical Oceanography, Recife, PE. 34:12-30.

Carvalho-Filho, A. 1999. Peixes: Costa Brasileira. $3^{\circ}$ Edição. São Paulo, Melro, 239p.

Cowen, R. K.; Hare, J. A. \& Fahay, M. P. 1993. Beyond hydrography. Can physical processes explain larval fish assemblage within the middle Atlantic bight? Bulletin of Marine Science. 53:567-587.

Cowen, R. K. 2002. Larval dispersal and retention and consequences for population connectivity. In: P.F. Sale (ed.) Ecology of Coral Reef fishes: Recent Advances. Academic Press. 149-170pp.

Dempster, T.; Gibbs, M. T.; Rissik, K. D. \& Suthers I. M. 1999. Beyond hydrograph: Daily ichthyoplankton variability and short term oceanographic events on the Sydney Continental Shelf. Continental Shelf Research. 17:1461-1481.

Davis, T. L. \& West, G. J. 1993. Maturation, reproductive seasonality, fecundity, and spawning frequency in Lutjanus vittus (Quoy and Gaimard) from the North Shelf of Australia. Fishery Bulletin. 91:224-236.

Doyle, M. J.; Morse, W. W. \& Kendall,Jr., A. W. 1993. A comparision of larval fish assemblages in the temperate zone of northeast Pacific and the northwest Atlantic ocean. Bulletin of Marine Science. 53:588-644.

Ekau, W.; Westhaus-Ekau, P. \& Medeiros, C. 1999. Large scale distribution of fish larvae in the continental shelf waters off North-East Brazil. Archive of Fishery and Marine Research. 47:183-200.

Ekau, W. \& Knoppers, B. 1999. An introduction to the pelagic system of the north-east and east Brazilian shelf. Archive of Fishery and Marine Research. 47:113-132.

Ekau, W.; Westhaus-Ekau, P.; Macêdo, S. J. \& Dorrien, C. V. 2001. The larval fish fauna of the "Canal de Santa Cruz" estuary in Northeast Brazil. Tropical Oceanography. 29:117-128.

Eppley, R. W. \& Weiler, C. S. 1979. The dominance of nanoplankton as an indicator of marine pollution: a critique. Oceanologica Acta. 2:241-245.

Eskinazi-Leça, E. \& Koening, M. L. 1986. Diatoms (Bacillariophyceae) distribution in the Suape area (Pernambuco-Brazil). Trabalhos Oceanográficos da Universidade Federal de Pernambuco. 19:73-100.
Evans, D. L. \& Signorini, S. S. 1985. Vertical structure of the Brazil current. Nature. 315:48-50.

Ferreira, C. E. L. \& Gonçalves, J. E. A. 1999. The unique Abrolhos reef formation (Brazil): need for specific management strategies. Coral Reefs. 18:1-352.

Govoni, J. J. 1993. Flux of larval fishes across frontal boundaries: Examples from the Mississippi river plume front and the western gulf stream front in winter. Bulletin of Marine Science. 53:538-566.

Heath, M. R. 1992. Field investigations of the early life stages of marine fish. Advances in Marine Biology. 28:1-174.

Humann, P. \& Deloach, N. 2002. Reef fish identification: Florida, Caribbean and Bahamas. Jacksonville, New World Publications, 481p.

Katsuragawa, M. 1990. Estudo sobre o desenvolvimento, a distribuição e a abundância de larvas de carangídeos da costa sudeste do Brasil. Tese de Doutorado. Universidade de São Paulo. 155p.

Leis, J. M. \& Remmis D. S. 1983. The larvae of IndoPacific coral reef fishes. Sydney, New South Wales University Press, 269p.

Leis, J. M. \& Goldman, B. 1987. Composition and distribution of larval fish assemblages in the Great Barrier Reef lagoon, near Lizard Island, Australia. Australian Journal of Marine and Freshwater Research. 38:211-223.

Leis, J. M. 1991. The pelagic stage of reef fishes: the larval biology of coral reef fishes. In: P.F. Sale (ed.) The ecology of fishes on coral reefs. San Diego, Academic Press. 183-230pp.

Leis, J. M. \& Mccormick, M. I. 2002. The biology, behaviour and ecology of the pelagic, larval stage of coral reef fishes. In: P.F. Sale (ed.) Coral Reef Fishes. Elsevier Science. 171-199pp.

Mafalda Jr, P. \& Silva, V. R. F. 1996. Caracterização do ictioplâncton do sistema estuarino-lagunar de Jequiá, Alagoas. Boletim de Estudos de Ciências do Mar. 9:89-104.

Marcolin, C. R.; Conceição, B. L.; Nogueira, M. M.; Mafalda Jr, P. \& Johnson, R. 2010. Mesozooplankton and ichthyoplankton composition in two tropical estuaries. Check List. 6(2):210-216.

Medeiros. C.; Araújo, M.; Rolinic, M. \& Freitas, I. 2009a. Estrutura termohalina da região oeste do Atlântico Tropical - ZEE/NE. In: F. H. V. Hazin (Ed.) Meteorologia e Sensoriamento Remoto, Oceanografia Física, Oceanografia Química, Oceanografia Geológica. Programa REVIZEE- Score Nordeste, Vol 1. Fortaleza, Martins \& Cordeiro Ltda. 40-55pp.

Medeiros. C.; Araújo, M.; Rolinic, M. \& Freitas, I. 2009b. Massas de água da região oeste do Atlântico Tropical-ZEE/NE. In: F. H. V. Hazin (Ed.) Meteorologia e Sensoriamento Remoto, Oceanografia Física, Oceanografia Química, Oceanografia Geológica. 
Programa REVIZEE-Score Nordeste, Vol 1. Fortaleza, Martins \& Cordeiro Ltda. 56-69pp.

Meekan, M. G.; Carleton, J. H.; Mckinnon, A. D. \& Flynn, K. M. 2003. What determines the growth of tropical reef fish larvae in the plankton: Food or temperature? Marine Ecology Progress Series. 256:193-204.

Menzel, D. W. \& Rhyther, J. H. 1961. Nutrients limiting the production of phytoplankton in the Sargasso Sea with especial reference to iron. Deep Sea Research. 7:276-281.

Moura, R. L.; Figueiredo, J. L. \& Sazima, I. 2001 A new parrotfish (Scaridae) from Brazil, and revalidation of Sparisoma amplum (Ranzani, 1842), Sparisoma frondosum (Agassiz, 1831), Sparisoma axillare (Steindachner, 1878) and Scarus trispinosus (Valenciennes, 1840). Bulletin of Marine Science. 68:505-524.

Neumann-Leitão, S.; Gusmão, L. M.; Silva, T.; Nascimento-Vieira, D. A. \& Silva, A.P. 1999. Mesozooplâncton biomass and diversity in coastal and oceanic waters off North-Eastern Brazil. Archive of Fishery and Marine Research. 47:153-165.

Nobrega F. M.; Lessa, R. \& Santana, F. M. 2009. Peixes Marinhos da Região Nordeste do Brasil. In: F. H. V. Hazin (Ed.) Programa REVIZEE-Score Nordeste, Vol 6. Fortaleza, Martins \& Cordeiro Ltda. 1-203pp.

Nonaka, R. H.; Matsuura, Y. \& Susuki, K. 2000. Seasonal variation in larval fish assemblages in relation to oceanographic conditions in the AbroIhos Bank region off eastern Brazil. Fishery Bulletin. 98:767-784.

Omori, M. \& Ikeda, T. 1984. Methods in marine zooplankton ecology. New York, John wiley \& Sons, $332 p$.

Owen, R. W. \& Zeitschel, B. 1970. Phytoplankton production: seasonal changes in the ocean eastern tropical Pacific. Marine Biology. 7:32-36.

Passavante, J. Z. O. \& Feitosa, F. A. N. 1995. Produção primária do fitoplâncton da plataforma continental de Pernambuco (Brasil): área de Piedade. Boletim Técnico Científico do CEPENE. 3:7-22.

Peterson, R. G. \& Stramma, L. 1991. Upper-level circulation in the South Atlantic Ocean. Progress in Oceanography. 26:1-75.

Reid, J. L. 1989. On the total geostrophic circulation of the South Atlantic Ocean: Flow, pattern, tracers and transport. Progress in Oceanography. 23:149-244.

REMAC. 1975. Mapas batimétricos da margem continental brasileira. In: Projeto REMAC-PETROBRÁS. Rio de Janeiro, Série Projeto REMAC- Mapas.
Richards, J. W. 2006. Early Stages of Atlantic Fish An Identification guide for the Western Central North Atlantic. Taylor \& Francis Group, 2640p.

Ryther, J. H. \& Menzel, D. W. 1965. On the production, composition and distribution of organic matter in the western Arabian Sea. Deep Sea Research. 12:199209.

Sampey, A.; Meekan, M. G,; Carleton, J. H.; Mckinnon, A. D. \& Mccormick M. I. 2004. Temporal patterns in distributions of tropical fish larvae on the North-west shelf of Australia. Marine and Freshwater Research. 55:473-487.

Smith, P. E. \& Richardson, S. L. 1977. Standart techniques for pelagic fish eggs and larvae surveys. FAO Fisheries Technical Paper. 75:1-100.

Stramma, L. 1991. Geostrophic transport of the South Equatorial Current in the Atlantic. Journal of Marine Research. 49:281-294.

Streelman, J. T.; Alfaro, M.; Westneat, M. W.; Bellwood, D. R. \& Karl, S. A. 2002. Evolutionary history of the parrotfishes: biogeography, ecomorphology, and comparative diversity. Evolution. 56:961-971.

Strickland, J. D. H. \& Parsons, T. R. 1972. A pratical handbook of seawater analysis. Bulletin of Fisheries Research Board of Canada. 167:1-311.

Tanaguchi, A. 1972. Geographical variation of primary production in the western Pacific Ocean and adjacent seas with reference to the interrelation between various parameters of primary production. Mem. Faculty of Fisheries Hokkaido University. 19:1-34.

Tchernia, P. 1980. Descriptive Regional Oceanography. Oxford, Pergamom Press, 253p.

Thomas, W. H. 1966. Surface nitrogenous nutrients and phytoplankton in the north-eastern tropical Pacific Ocean. Limnology and Oceanography. 11:393-400.

Travassos, P.; Hazin, F. H. V.; Zagaglia, J. R.; Advíncula, R. \& Schober, J. 1999. Thermohaline structure around seamounts and islands off North-Eastern Brazil. Archives of Fisheries and Marine Research. 47(2/3): 211-222.

Whitfield, A. K. \& Paterson, A. W. 2003. Distribution patterns of fishes in a freshwater deprived Eastern Cape estuary, with particular emphasis on the geographical headwater region. Water SA. 29:6167.

Young, P. C. J. M. \& Leis, H. F. 1986. Seasonal and spatial distribution of fish larvae in waters over the northwest continental shelf of Western Australia. Marine Ecology Progress Series. 31:209-222.

Submetido: Outubro/2008 Revisado: Julho/2009 Aceito: Agosto/2010 